\title{
Feasibility of Afforestation as an Equitable Nature-Based Solution in Urban Areas
}

T. Chakraborty ${ }^{1 *}$, T. Biswas ${ }^{2 *}$, L. S. Campbell ${ }^{3}$, B. Franklin ${ }^{2}$, S.S. Parker ${ }^{4}$, M. Tukman ${ }^{5}$

${ }^{2}$ California Program, The Nature Conservancy, Sacramento, CA, USA

${ }^{3}$ Contour Group, Salt Lake City, UT, USA

${ }^{4}$ California Program, The Nature Conservancy, Los Angeles, CA, USA

${ }^{5}$ Principal, Tuckman Geospatial Analysis, LLC, Santa Rosa, CA, USA

Corresponding Authors: T. Chakraborty (tc.chakraborty@yale.edu) and T. Biswas (tanushree.biswas@tnc.org)

This preprint is for a manuscript currently under review. Note that the content may change somewhat in subsequent versions of the manuscript. Please feel free to contact any of the authors if you have any feedback or suggestions 
Abstract

Keywords: Nature-based solutions, urban afforestation, environmental disparities, climate adaptation, urban planning important to quantitatively assess the feasibility of such strategies to utilize space efficiently and prioritize lower-income communities, who have fewer options for climate change adaptation. Here we combine data from US census estimates, satellites, and satellite-derived products to develop a framework to target potentially suitable areas for urban afforestation to mitigate urban heat and minimize tree cover disparity. We test this framework for California, the most populated state in the US and the 5th largest economy (by GDP) in the world, and show that space exists for an additional 34 million (1.2 million acres of) trees in the state's urban areas. This would reduce the average urban land surface temperature (LST) by $1.7^{\circ} \mathrm{C}$ and provide multiple co-benefits totaling $\$ 1.1$ billion annually, including reduction in heat-related medical visits ( $>3000$ over 10 years) and 3.9 million metric tons of annual $\mathrm{CO}_{2}$ sequestration. Without any intervention to reduce urban LST, the net present value of the social cost of carbon from residential electricity use ranges from $\$ 12.9$ million to $\$ 102.1$ million. Because funding is limited, we provide suitability scores for urban afforestation at the census block group (CBG) scale based on multiple considerations. In California for instance, equitable urban afforestation in CBGs with positive suitability scores will serve $89 \%$ of the $\approx 9$ million urban residents in the lowest income quartile for their cities. This method can guide equitable urban afforestation efforts and can be scaled to other North American cities.

\section{Funding: Conservation Technology Innovation Fund, The Nature Conservancy}


Modern cities are centers of social, economic, and cultural activities and house over half the global population (Lewis and Maslin, 2015; Nations, 2018). The higher temperature in cities compared to surrounding areas, usually a consequence of the replacement of natural surfaces with built-up areas, is associated with increases in heat-related mortality and morbidity and higher cooling energy demand at the urban scale (McMichael et al., 2008; Oke, 1982; Santamouris, 2014). With both the proportion of humans residing in cities and urban temperatures expected to increase in the future, urban areas have to be at the forefront of climate change adaptation and mitigation ("Cities must protect people from extreme heat," 2021). These adaptation and mitigation strategies must be deployed at the sub-urban scale because urban areas have large spatial heterogeneity, with disproportionately higher heat-related impacts on vulnerable communities (Chakraborty et al., 2019; Harlan et al., 2006). For instance, in the US, urban land surface temperature (LST) is generally higher in lower income neighborhoods and is strongly associated with disparities in urban tree cover (Benz and Burney, 2021; Chakraborty et al., 2020; Hoffman et al., 2020; Hsu et al., 2021; McDonald et al., 2021; Nesbitt et al., 2019). These vulnerable populations have fewer options for dealing with heat extremes that contribute to over 5 million deaths a year globally (Zhao et al., 2021). Of the many urban heat mitigation strategies proposed, afforestation (defined as the planting of trees and creation of forest where it was historically absent) is a nature-based solution with multiple co-benefits, and if implemented strategically, would sequester carbon, moderate air pollution, reduce energy demand, moderate health impacts during hot summer months, and address additional environmental disparities (Dorst et al., 2019; Fargione et al., 2018; McDonald et al., 2020; McPherson et al., 2017; Remme et al., 2021).

Although there are numerous studies on associations between vegetation cover and local temperatures (Augusto et al., 2020; Chakraborty and Lee, 2019; Zhou et al., 2016; Ziter et al., 2019), we know less about the physical and logistic viability of using afforestation to strategically mitigate urban heat and address disparities in urban green space (Drescher, 2019; Ziter et al., 2019). Without an intentional effort to reduce urban heat in the most impacted communities, we would leave these vulnerable populations exposed to the dire consequences of high urban temperatures, which will be further exacerbated by global and regional climate change. About $85 \%$ of Americans live in metropolitan areas. High population densities, presence of built-up areas, and the necessity for critical infrastructure all limit the plantable area for new trees within urban areas. This component is critical to consider for future urban planning and is missing in the existing literature. Many multi-city studies are based on model simulations, which have simplified or no representation of urban vegetation, and cannot sufficiently resolve intra-urban variability due to computational bottlenecks and scale limitations of physical parameterizations (Grimmond et al., 2011; Zhao et al., 2017; Zheng et al., 2021). The availability of spatially continuous satellite observations provides an opportunity to develop a scalable framework that constrains heat mitigation and other benefits of urban afforestation.

Here we combine medium to high-resolution satellite-derived estimates of LST and tree cover with several ancillary inputs, including US census estimates, to develop a suitability algorithm that frames the efficiency of urban afforestation as an equitable nature-based solution to address urbanscale climate change. Our conceptual framework is applied over more than 200 urban areas in California, the most populated state in the US and the 5th largest economy (by GDP) in the world, which has seen increased susceptibility to heatwaves due to climate change (Hulley et al., 2020). This method, developed at the census block group (CBG) level, uses publicly available data and is designed to be scaled up across other North American cities. Leveraging the wealth of data and literature on the benefits of tree cover in California, we quantify some of the potential co-benefits of urban afforestation, as measured through reductions in heat-related health outcomes, energy used 
for cooling, and increases in carbon sequestration. It is becoming increasingly clear that climate change requires multi-pronged mitigation strategies that can be applied across scales. Because urban areas suffer from local-scale environmental concerns that affect a large proportion of human residents, strategic and novel urban policies have the potential to re-design urban areas for climate change resilience while simultaneously sequestering carbon and furthering a more equitable distribution of environmental resources. The results of this study can provide policymakers a necessary tool to achieve these goals and strategically benefit low-income and frontline communities. While our current work only focuses on California, in the future, the framework developed here can be improved and expanded across North American cities, counties, and states to spatially assess optimal locations for urban afforestation.

\section{Materials and Methods}

\subsection{Regions of interest and summarizing physical and socioeconomic data}

We develop the conceptual suitability framework for urban areas in California, where high temperature is a significant public health concern. The urban boundaries are based on the US Census Bureau's urbanized area dataset, which includes 211 boundaries that intersect with the state border ("2010 Census Urban and Rural Classification and Urban Area Criteria,"). Of these, some of the boundaries are primarily in Nevada and Arizona, and since the EarthDefine data ("EarthDefine,"), used to estimate current canopy cover, were not available outside California, these were removed from the calculations. We also remove the city of Paradise from our analysis since the city was burned in a wildfire in 2018. The final selection of 202 boundaries is intersected with CBG polygons. We use CBGs since they are the finest level of geographic aggregation for which median household income is publicly available. CBG level population, income, and number of housing units for 2018 from the American Community Survey (ACS) (Mather et al., 2005) are extracted using the census API package for the R programming language.

For each CBG, we also calculate the area of land based on the $30 \mathrm{~m}$ National Land Cover Database (NLCD) for 2016 (Wickham et al., 2021). Similarly, the fraction of land that is urban is based on the sum of the area of low, medium, and high intensity urban classes in the NLCD dataset. The current tree cover for each CBG is calculated from the $1 \mathrm{~m}$ EarthDefine product, which uses deep learning algorithms to map urban tree cover in California ("EarthDefine,"). Since EarthDefine data are only available within the urban boundaries, the portion of any CBG crossing the boundaries is masked out for all analyses.

\subsection{Estimating land surface temperature}

Satellite observations can provide spatially continuous estimates of LST, which is important for studying intra-urban variability (Benz et al., 2021; Duguay-Tetzlaff et al., 2015; Gallo et al., 1995). Although this radiometric surface temperature is not physically identical to near-surface air temperature, urban areas rarely have dense meteorological networks to estimate spatial variability of air temperature (Muller et al., 2013). Here we use daytime (at roughly $\approx 10: 20$ am local time) LST derived from the Landsat 5 satellite (Loveland and Dwyer, 2012), which provides observations in the thermal band at a native resolution of $120 \mathrm{~m}$. Landsat 5 measures top of the atmosphere thermal radiance, which needs to be converted into LST. This conversion is done here using the Statistical Mono-Window (SMW) algorithm based on the linearization of the radiative transfer equation (Malakar et al., 2018). The equation can be formulated as:

$\mathrm{LST}=A_{i} \frac{L_{\text {sen }}}{\varepsilon}+B_{i} \frac{1}{\varepsilon}+C_{i}$

Here $L_{\text {sen }}$ is the top of the atmosphere thermal radiance measured by the sensor in a particular thermal band (in this case, between 10.4 and 12.5 micron) and $\varepsilon$ is the surface emissivity for the same wavelength band. $A_{i}, B_{i}$, and $C_{i}$ are empirical coefficients determined from radiative transfer 
calculations for 10 classes of columnar water vapor content in the atmosphere. The value of $\varepsilon$ is estimated for each pixel based on measurements by the Advanced Spaceborne Thermal Emission and Reflection Radiometer (ASTER) (Abrams, 2000), which is then adjusted using thresholds of Normalized Difference Vegetation Index (NDVI), a proxy for surface vegetation (Rouse et al., 1974). Using the version of the SMW algorithm implemented on the Google Earth Engine cloud computing platform (Gorelick et al., 2017) by Ermida et al. (2020), the 5-year mean annual, summertime (June-July-August), and wintertime (December-January-February) LST are calculated from 2007 to 2011 for California.

\subsection{Calculating surface urban heat island intensity}

We calculate the surface urban heat island (SUHI) intensity for each of the 202 selected urban areas in California at both the urban scale and at the CBG scale. For both scales, the rural reference is identical and is developed using an iterative buffering procedure around the urban boundary (Chakraborty et al., 2021a) using a step size of $30 \mathrm{~m}$. The final buffered area is approximately equal to the area of the urban area it surrounds.

The urban scale SUHI is calculated as the difference in mean LST (Eq. 2) between the urban pixels (medium + high intensity urban classes from the NLCD dataset) in the urban boundary (LST urb) and the non-urban and non-water (and water-adjacent) pixels in the reference buffer (LST $\mathrm{Lur}_{\text {r). }}$

$\mathrm{SUHI}=\mathrm{LST}_{\text {urb }}-\mathrm{LST}_{\text {rur }}$

On the other hand, the CBG scale $\mathrm{SUHI}\left(\mathrm{SUHI}_{\mathrm{i}}\right)$, where i represents an individual $\mathrm{CBG}$, is calculated as the difference in the mean urban LST of the pixels intersecting the CBG (LST urb,i) and the earlier computed LST of the reference buffer for the whole urban boundary. Since elevation strongly controls temperature, for the rural reference, care is taken to account for this by only selecting pixels that are within $50 \mathrm{~m}$ of the median elevation of the urban boundary based on the Global Multi-resolution Terrain Elevation Data (GMTED) (Danielson and Gesch, 2011).

Although the CBG scale results are primarily used for the suitability algorithm, the urban scale estimates are useful to check the accuracy of our methods. We compare the results for the urbanized areas in California $(n=57)$ with a recent nationwide dataset (Chakraborty et al., 2020). Overall, for all cases (annual, summertime, and wintertime), the variability in SUHI is captured well by our analysis (Fig. 1). The difference in magnitude is expected since the nationwide dataset uses the Simplified Urban Extent (SUE) algorithm (Chakraborty and Lee, 2019), provides more conservative estimates of SUHI than buffer-based methods and because Landsat-derived LST tends to be higher than those calculated from Moderate Resolution Imaging Spectroradiometer (MODIS) observations (Chakraborty et al., 2021b). 


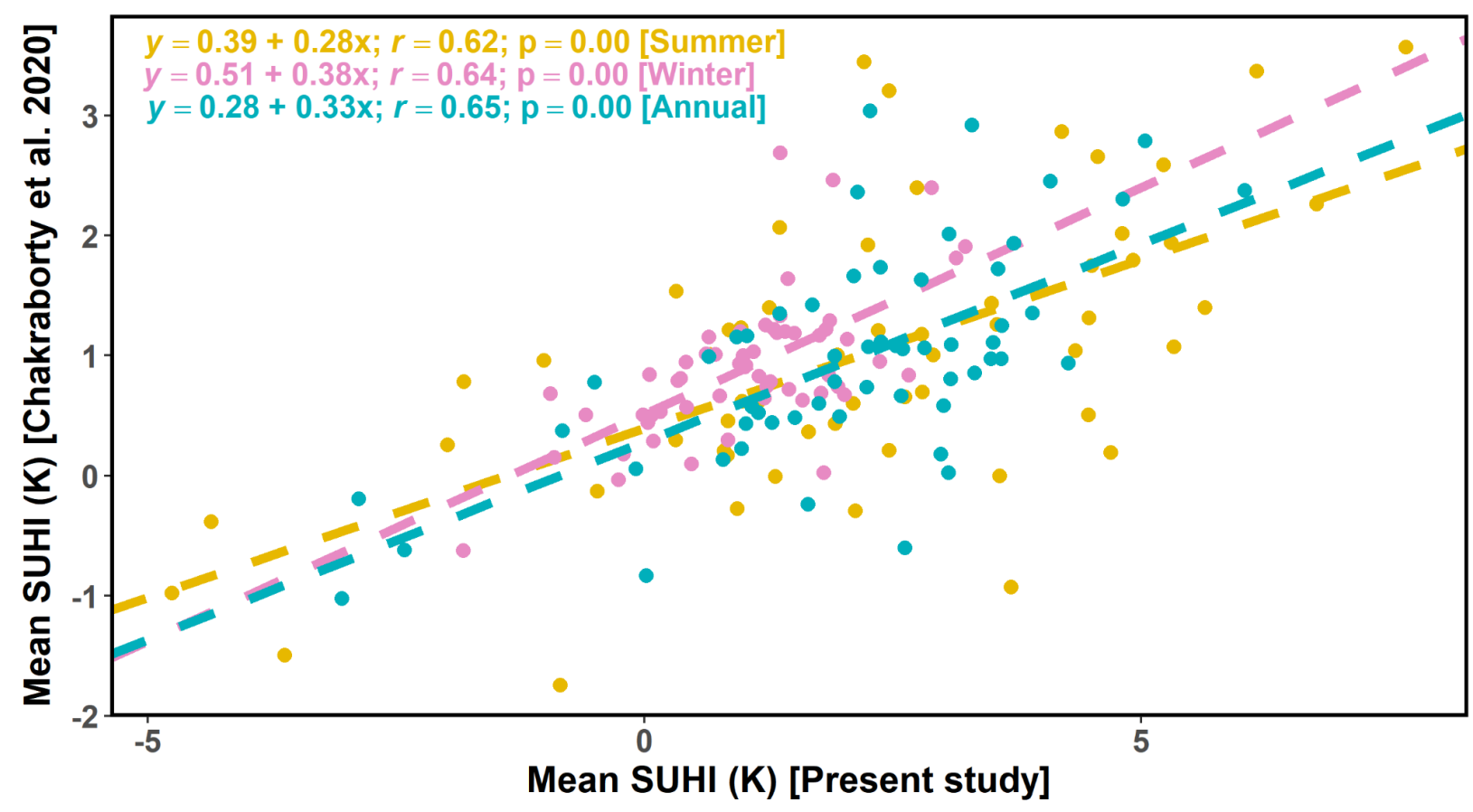

Fig. 1. Evaluation of calculated daytime SUHI for summer, winter, and entire year derived from Landsat data for the present study for the 57 urbanized areas in California against a previous nationwide dataset (Chakraborty et al., 2020) derived from MODIS satellite observations.

\subsection{Computing plantable area for urban afforestation}

We refer to the tree planting within Californian cities as afforestation and not reforestation since a lot of these regions did not originally have forests. We estimated the potential area for this afforestation (or the plantable area) for each CBG from the total empty space (area that is not built up, not impervious, and not vegetated) within $\mathrm{a} \approx 900 \mathrm{~m}^{2}$ Landsat pixel. This was done by removing the total area of NLCD 2016's low, medium and high-intensity developed pixels and as well as the total EarthDefine tree-covered area from the total area of each CBG. Areas extending beyond census-block tracts, exurban areas and impervious surfaces (such as buildings or parking lots) were also excluded. The area of afforestation is converted into number of trees using the mean tree density for low density residential areas in Los Angeles and Sacramento (70 per hectare or 28.33 per acre) found in McPherson et al. (McPherson et al., 2013).

\subsection{Estimating surface urban heat island mitigation potential}

A multivariate linear regression was developed for each urban area to quantify the relationship between percentage tree canopy cover (Can) and SUHI. Although this relationship was the primary focus of the regression, physical characteristics other than tree cover - from building density and height to overall urban form to the distribution of vegetation - also influence SUHI (Liu et al., 2021; Zhou et al., 2016). We did not have these relevant uniform California-wide datasets available. Therefore, we incorporated ancillary information to serve as a proxy for some of the desired information about the physical environment. The additional variables we chose to use include: distance from the coast (Dist ${ }_{C o a}$ ), distance from the centroid of the urban area (Disturb), population density (Pop), income (Inc), and the percentage of the area covered by NLCD high intensity developed (NLCDHigh), medium intensity developed (NLCD ${ }_{\mathrm{Med}}$ ), low intensity developed

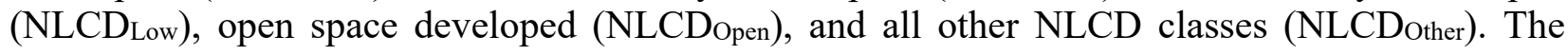
linear model can be formulated as: 
$y=\beta_{0}$ Can $+\beta_{1}$ Dist $_{\text {Coa }}+\beta_{2}$ Disturb $+\beta_{3}$ Pop $+\beta_{4}$ Inc $+\beta_{5} \mathrm{NLCD}_{\text {High }}+\beta_{6} \mathrm{NLCD}_{\text {Med }}+\beta_{7} \mathrm{NLCD}_{\text {Low }}+$ $\beta_{8} \mathrm{NLCD}_{\text {Open }}+\beta_{9} \mathrm{NLCD}_{\text {Other }}+\beta_{10}$

where $\beta_{0}$ to $\beta_{10}$ are the coefficients and $y$ is the daytime summer SUHI intensity.

Since these additional variables are assumed not to change with the addition of tree canopy cover, we can use this equation to estimate the sensitivity of SUHI to Can from the slope of the first term in the regression $\left(\beta_{0}\right)$. This slope was used to calculate the SUHI change $(\Delta \mathrm{UHI})$ for the three tree canopy cover scenarios (MPUA, TREEGAP, and UHIGAP) outlined in the next subsection. See an example of this multi-variate linear model in Fig. 2 below.

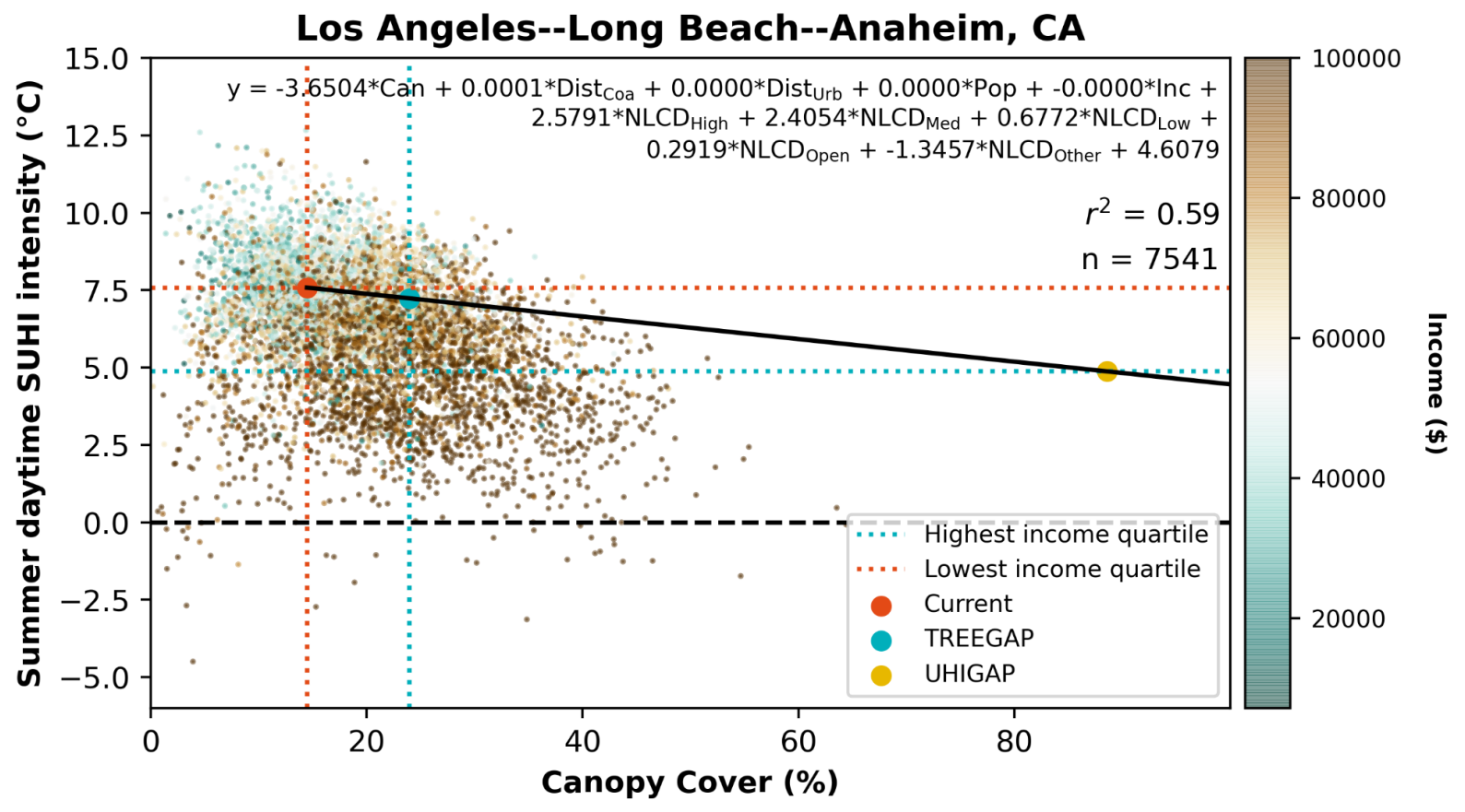

Fig. 2 An example of the linear model used to represent SUHI as a function of census and satellitederived data for the urban cluster encompassing Los Angeles, Long Beach, and Anaheim. The SUHI reduction is calculated for the different scenarios using this model and estimates of plantable area within CBGs. The mean canopy cover percentage and SUHI corresponding to the highest and lowest income quartile CBGs in the urban cluster are also shown.

The $r^{2}$ for each city's regression ranged from 0.25 to 1.86 cities have $r^{2}$ greater than or equal to 0.5 . Of these, 81 cities have a slope $\left(\beta_{0}\right)$ less than 0 in the multi-variate linear model, ranging from -28.98 to -0.57 . Cities with $r^{2}$ less than 0.3 and fewer than 15 CBGs were disregarded for regressionrelated analysis in this paper. Only in 109 of 21358 CBGs $(0.5 \%)$ is SUHI after afforestation greater than current SUHI due to statistical artifacts, which include uncertainty in the input data and not being able to fully resolve the coastal influence on SUHI. These are also ignored when summarizing the results.

\subsection{Designating priority census block groups for urban afforestation}

Our prioritization approach follows a stepwise analysis, depicted in Fig. 3, that determines:

i) How many acres of trees are needed to close the tree gap (difference in tree cover between the highest income quartile CBGs and the CBG of interest; TREEGAP) and SUHI gap (difference in daytime summer SUHI between the highest income quartile CBGs and the CBG of interest; UHIGAP)?

ii) How much plantable area is available for urban afforestation? 
224 iii) What is the maximum SUHI reduction potential if we maximized canopy (Maximum Potential 225 Urban Afforestation or MPUA scenario; $\triangle$ UHIMPUA)?

226 iv) What is the SUHI reduction potential if we only closed the tree gap and SUHI gap (TREEGAP 227 and UHIGAP scenarios, respectively)?

228 v) Optimize the benefit towards high population density and low household income blocks through 229 implementation of suitability scores.

230 Unlike the MPUA scenario, which represents an upper bound for potential urban afforestation, the 231 TREEGAP and UHIGAP scenarios are intended to minimize disparities in urban tree cover and 232 daytime SUHI, respectively, by selectively targeting CBGs with vulnerable populations. For the 233 UHIGAP scenario, the sensitivity of SUHI to tree cover percentage is computed for each city using 234 Eq. 3. For the TREEGAP and UHIGAP scenarios, if the potential area was less than required to 235 close the tree canopy cover gap and SUHI gap, respectively, the potential area (for MPUA scenario) 236 was used instead of the area needed to close the gaps. 


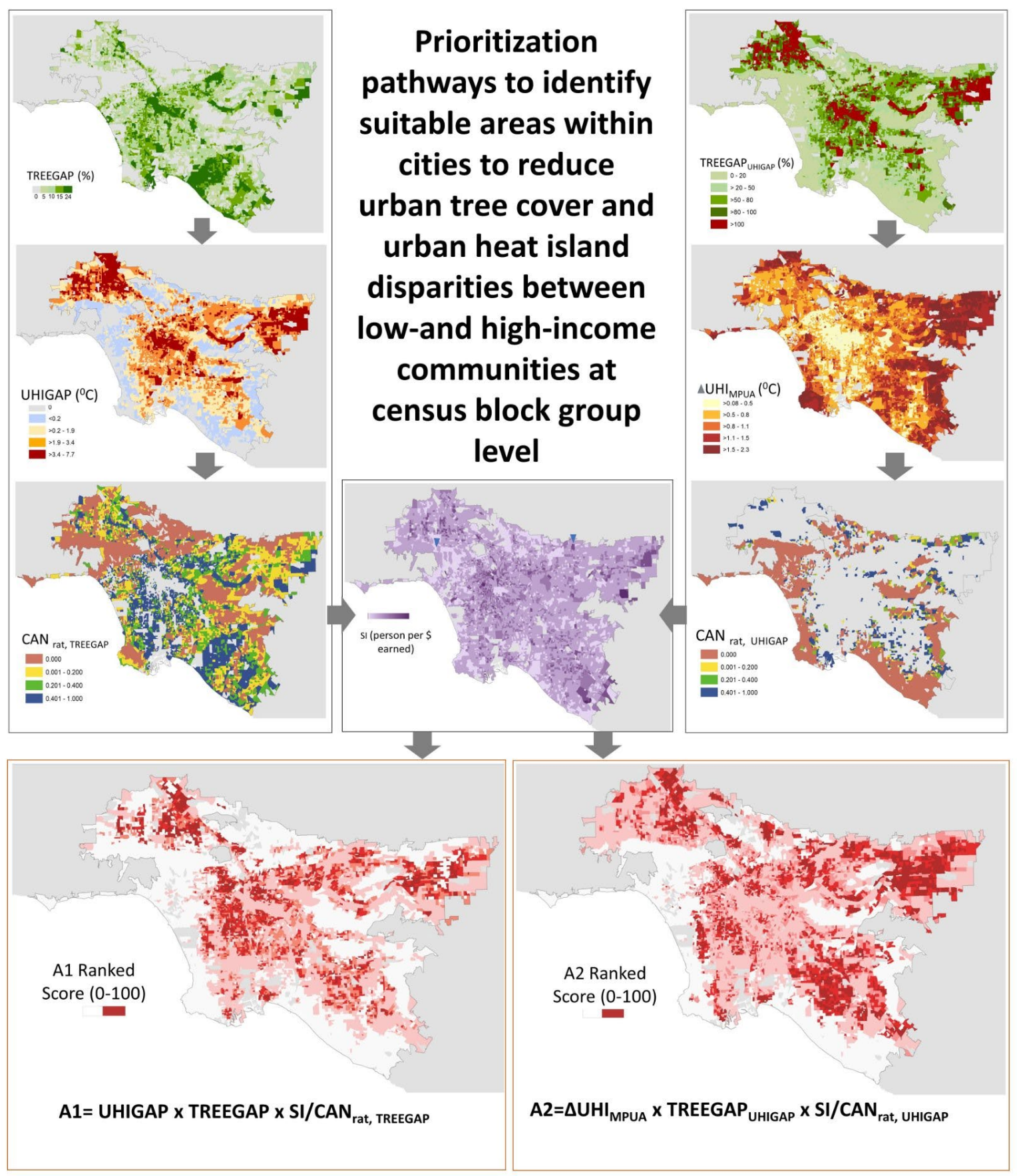

Fig. 3 Summary of workflow to calculate A1 and A2 suitability scores for each urban CBG in California to close tree cover and urban heat island disparity between low-and high-income communities. Intermediate results for Los Angeles are shown for illustrative purposes. SI is the sensitivity index for the CBG calculated by dividing the population by income. A1 is the suitability score to reduce urban tree cover disparity. Here $\mathrm{CAN}_{\text {rat, TREEGAP }}$ is the ratio of additional canopy cover needed to meet the TREEGAP scenario vs. the amount of available space. A2 is the suitability score to close urban heat island disparity. It identifies CBGs with the most potential area for afforestation and the most need of additional canopy cover to close the UHIGAP, where $\triangle$ UHIMPUA is the expected change in daytime summer SUHI for the MPUA scenario, TREEGAPUHIGAP is the

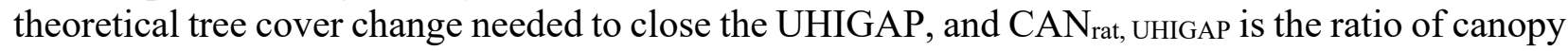
cover for the UHIGAP and MPUA scenarios. See Methods for more details. 
We designate $\mathrm{CBGs}$ into $\mathrm{A} 1$ and $\mathrm{A} 2$ priority block groups following two prioritization pathways. Both A1 and A2 include a sensitivity index (SI) that identifies the high priority CBGs with high population and low income:

SI = Population / Income

The A1 score, which focuses on identifying CBGs with a high UHIGAP, high TREEGAP, and sufficient potential area to meet the TREEGAP goals, is calculated for all CBGs with a positive TREEGAP (e.g. have a lower tree canopy cover than the highest income quartile) using the equation:

A1 $=$ UHIGAP $*$ TREEGAP $*$ SI $/ \mathrm{CAN}_{\text {rat, }}$ TREEGAP

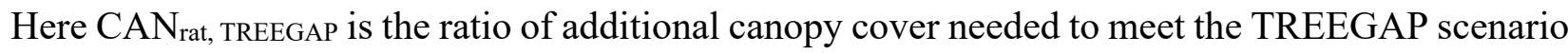
vs. the amount of available space.

The A2 score, which identifies CBGs with the most potential area for afforestation and the most need of additional canopy cover to close the UHIGAP, is calculated for all CBGs with a positive UHIGAP (e.g. have a higher SUHI than the highest income quartile) as:

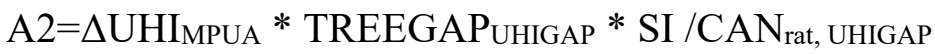

where $\triangle U_{H I} I_{M U A}$ is the expected change in daytime summer SUHI for the MPUA scenario, TREEGAPUHIGAP is the theoretical tree cover change needed to close the UHIGAP, and CAN rat, $_{\text {, }}$ UHIGAP is the ratio of canopy cover for the UHIGAP and MPUA scenarios. Following these formulations, all CBGs included in the calculation for each urban area are then ranked from 0 - 100 for both $\mathrm{A} 1$ and A2, with 100 being the most suitable for afforestation.

\subsection{Examining benefits and co-benefits of urban afforestation}

We estimate potential benefits and co-benefits of urban afforestation to better quantify the importance of such nature-based solutions beyond surface urban heat island mitigation. For this analysis, California is an ideal location due to widespread data availability, the state's susceptibility to heatwaves, and a rich scientific literature on the impact of urban afforestation (Chen et al., 2020; Hulley et al., 2020; McPherson et al., 2017; Shonkoff et al., 2011). While there are additional benefits to biodiversity, groundwater recharge, etc., we primarily focused on addressing tree cover inequality while benefiting the vulnerable populations exposed to excess urban heat and climate change ${ }^{16}$. Following our prioritization approach, we provide a summary of total benefits to health, energy savings and climate from carbon sequestered based on this intervention across the city as well as the state.

\subsubsection{Avoided heat-related health outcomes}

We calculate baseline values for the expected avoidance of heat-related health outcomes for a group of select urban areas in California by combining multiple health outcome datasets with summertime LST estimates (Fig. S1). The health outcome data include Emergency Department and Patient Discharge Datasets from the State of California, Office of Statewide Health Planning and Development (OSHPD), Multiple Cause of Death Files from the State of California, Department of Public Health, Office of Vital Statistics, and the Death Statistical Master File from Department of Public Health, Office of Vital Statistics. These data sources are combined to provide heat-related emergency department visits, hospitalizations, and deaths for 2009-2018 by patient zip code. For zip codes with less than 12 cases, the data are suppressed due to Health Insurance Portability and Accountability Act (HIPAA) privacy regulations. For these zip codes, we make a conservative estimate that the number is the minimum possible, i.e. 1 during the entire period. In parallel, we 
calculate the mean summertime LST during the study period (2007 - 2011) for each of those zip codes. Using this database, we calculate the sensitivity of heat-related health outcome (HO) per capita to summertime LST for all cities where the number of available zip codes exceed 10 using a linear model. This sensitivity $\left(\frac{\partial H O}{\partial L S T}\right)$ represents the number of heat-related health outcomes per capita for a unit change in LST. This includes 9 urban areas, namely Concord, Fresno, Los Angeles, Mission Viejo, Riverside, Sacramento, San Diego, San Francisco, and San Jose. We use this sensitivity, the daytime summer SUHI reduction for the MPUA scenario ( $\left.\triangle U H I_{M P U A}\right)$, and census population estimates (Pop) to calculate the avoided heat-related health visit $\left(\mathrm{HO}_{\mathrm{av}}\right)$ during a similar period using the equation:

$\mathrm{HO}_{\mathrm{av}}=\frac{\partial H O}{\partial L S T} \mathrm{Pop} \triangle \mathrm{UHIMPUA}$

A few caveats to note here. HO depends not only on LST (with air temperature being more relevant for heat-related health outcomes), but also on behavioural effects. We assume that the HO are primarily due to mean summertime temperature even though the HO dataset is available as a multiannual mean. In reality, a large fraction of these outcomes would be during extreme events, which are harder to predict from Landsat satellite observations. However, we assume that these extreme events add to already existing spatial variability in baseline LST, which is captured by our analysis. We would also not expect the sensitivity to HO to LST to be linear, meaning our estimates are mainly conservative. Given all these uncertainties, we stress that the estimates provided here should not be overanalyzed and are intended to support the importance of urban heat reduction on avoided heat-related health outcomes, which is also well established in cohort-based and physiological studies (Christidis et al., 2010; Hajat and Kosatky, 2010).

\subsubsection{Enhanced net carbon sequestration and afforestation cost-benefit}

We estimate the net carbon sequestration due to urban afforestation by taking the average of the values calculated by Nowak et al. (Nowak et al., 2013) for Los Angeles $\left(0.327 \mathrm{~kg} \mathrm{carbon} \mathrm{m}^{-2} \mathrm{yr}^{-1}\right)$, Sacramento $\left(0.221 \mathrm{~kg}\right.$ carbon $\left.\mathrm{m}^{-2} \mathrm{yr}^{-1}\right)$, and San Francisco $\left(0.107 \mathrm{~kg}\right.$ carbon $\left.\mathrm{m}^{-2} \mathrm{yr}^{-1}\right)$. We combine the average carbon sequestration rate of $0.218 \mathrm{~kg}$ carbon $\mathrm{m}^{-2} \mathrm{yr}^{-1}$ or $3.24 \mathrm{~kg} \mathrm{CO}_{2} \mathrm{acre}^{-1} \mathrm{yr}^{-1}$ with our estimated total urban afforestation potential to get the net carbon sequestration for each city. Note that this is a counterfactual analysis that assumes fully grown trees in the urban area. Moreover, we would assume a large degree of variability in this sequestration estimate based on the species of tree planted and other considerations like nutrient and water availability. This is a topic of continued research and given the scale we are working at, is beyond the scope of the present study.

To get bulk estimates for the cost and benefit for each afforestation scenario, we combine estimates of maximum tree density for urban areas in California with the mean annual cost and benefit per tree (\$19 for management and $\$ 47.83$ for benefit) for California's urban forests (McPherson et al., 2017).

\subsubsection{Reduced urban energy consumption}

The decrease in energy consumption during summer due to SUHI mitigation is primarily through reduced air conditioning needs. Here we estimate this decrease in energy consumption by combining estimates of urban $\mathrm{AC}$ saturation rate by California's Building Climate zones $(\mathrm{AC}, \mathrm{z})$, with the sensitivities of electricity consumption to ambient temperature $(T)$ and estimates of number of housing units $\left(H_{\mathrm{i}}\right)$ from the census data. The AC saturation rate is from Chen et al. (Chen et al., 2020) based on reported utility data throughout California. This includes central air conditioning, room AC, and evaporative coolers. The data are available for all but Climate zone 6. For this climate zone, we take the mean AC saturation for the whole state, which is 0.77 . Since Chen et al. (Chen et al., 2020) provided sensitivity values for various poverty percentiles, we take upper and lower 
337 bound estimates for the highest and lowest percentile bins in that study. For each CBG, the total

338 energy consumption reduction is formulated as:

$339 \quad \mathrm{E}_{\mathrm{red}, \mathrm{i}}=\frac{\partial E}{\partial T} H_{\mathrm{i}} \mathrm{AC}_{\mathrm{p}, \mathrm{z}}$

340 The energy saving values for individual cities are in Fig. S2.

$341 \quad 3$. Results

\subsection{Disparities in surface urban heat islands and tree cover in California}

Since SUHI is the difference in LST between a city and its rural background, by focusing on SUHI instead of actual temperature, we can isolate the urban contribution to local temperature and thus determine how effective urban-scale nature-based policies can be at addressing and resolving this local climatic impact of urbanization. Consistent with previous studies (Chakraborty et al., 2020; Imhoff et al., 2010), the daytime SUHI for cities in California is highest during summer (areaweighted mean and standard deviation across clusters of $2.42{ }^{\circ} \mathrm{C}$ and $3.03{ }^{\circ} \mathrm{C}$ ) and lowest in the winter $\left(0.92 \pm 1.27^{\circ} \mathrm{C}\right.$; Figs $4 \mathrm{a}, 4 \mathrm{~b}$, and 5$)$. Because summer has the highest potential for heatrelated mortality and morbidity, we focus on mitigating urban temperatures during this season. The city-mean summer daytime SUHI is positive for 164 of the 202 selected cities (higher than $5{ }^{\circ} \mathrm{C}$ in 36 cities) and negative, i.e. the rural background is relatively warmer, in 38 primarily arid cities (Fig. 4b). A negative SUHI (or urban cool island) over arid cities is generally due to additional tree cover and vegetation within the urban area versus its surroundings and is consistent with previous observational estimates (Chakraborty et al., 2020; Chakraborty and Lee, 2019; Imhoff et al., 2010). 

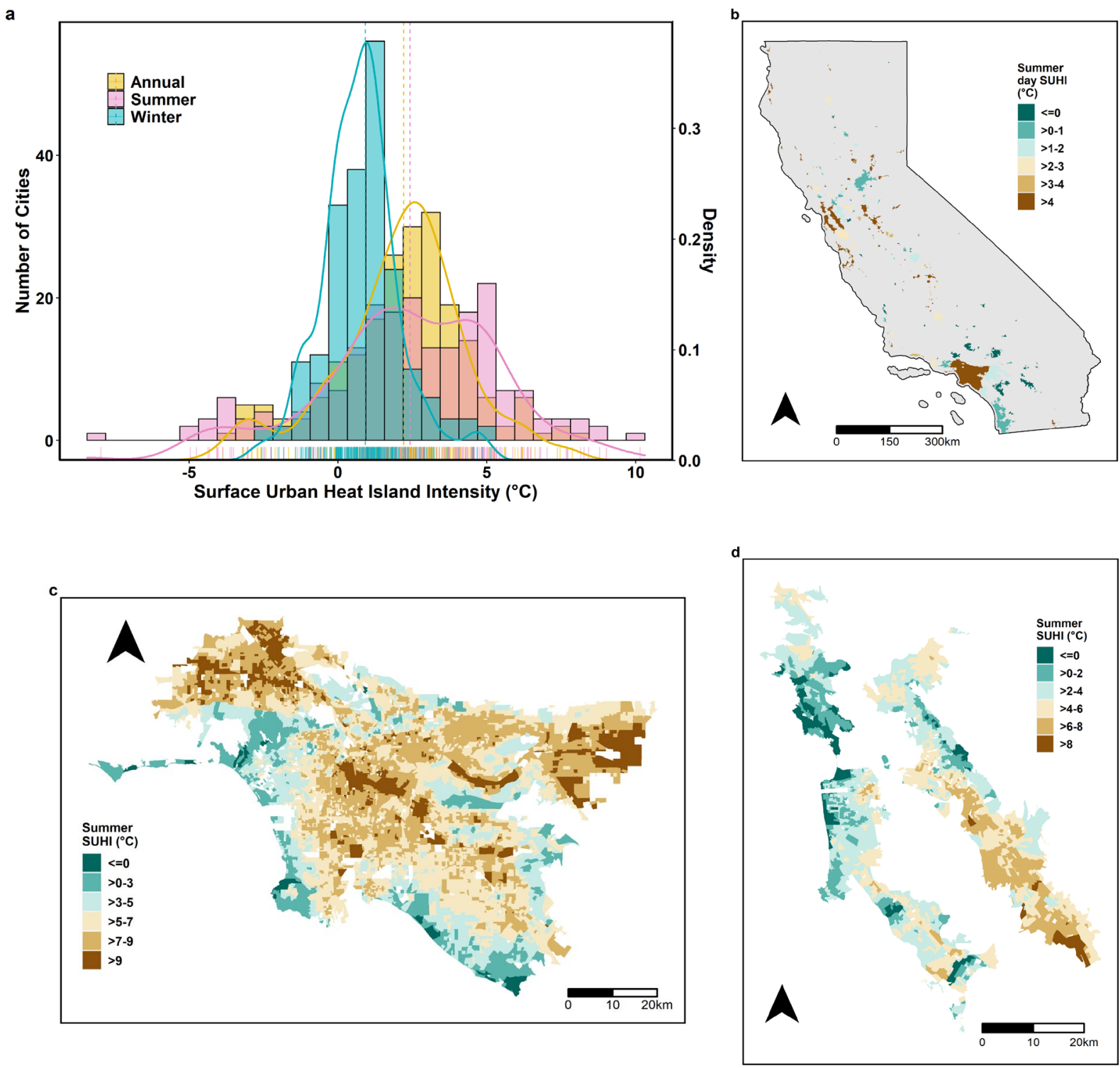

Fig. 4 Summary of daytime Surface Urban Heat Island (SUHI) intensity for California. Sub-figure (a) shows the statistical distribution of city-level mean annual, summertime, and wintertime SUHI in California during daytime based on satellite measurements from 2007 to 2011. Sub-figure (b) shows the statewide spatial distribution of city-level summer daytime SUHI, while sub-figures (c) and (d) show the intra-urban variability of SUHI for Greater Los Angeles and the San Francisco Bay area, respectively, at the CBG level. 

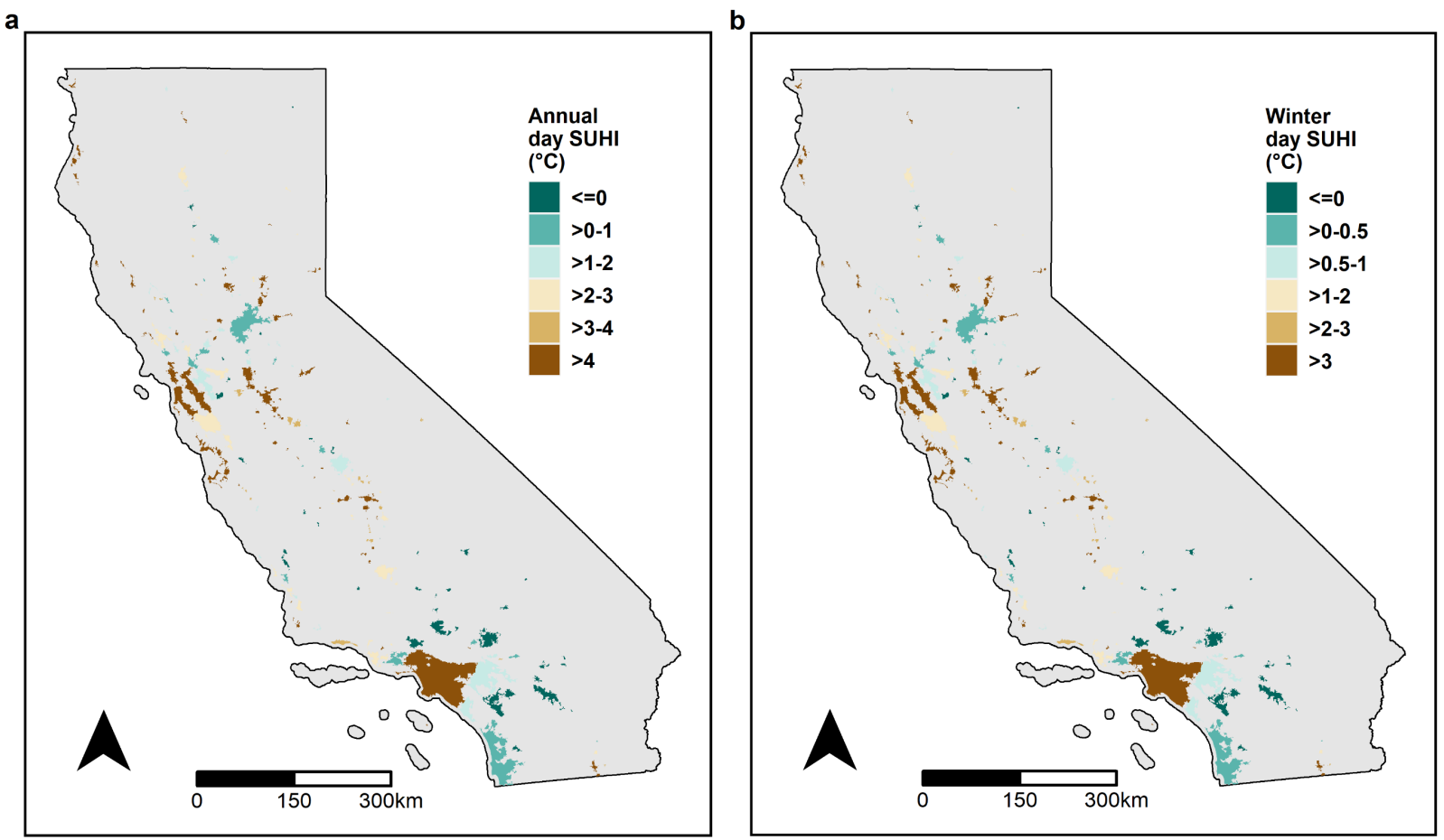

Fig. 5. Spatial distribution of city-level daytime SUHI. Sub-figures (a) and (b) show the spatial distribution of city-level annual and winter daytime SUHI, respectively.

The intra-urban variability in the daytime SUHI is large and can disproportionately impact lower income communities (Benz and Burney, 2021; Chakraborty et al., 2020, 2019; Hoffman et al., 2020; Hsu et al., 2021; Voelkel et al., 2018). We represent this variability by estimating SUHI at the CBG level, demonstrated for the Greater Los Angeles and San Francisco Bay areas (Figs 1c and 1d). We use a sample size threshold of at least 10 CBGs per city to test for linear relationships between variables (Fig. 6a) and find that over $89 \%$ of these cities (84 of 94) show negative associations between daytime SUHI and median income. Thus, in most cases, lower income populations live in regions with higher LST, with the composite mean correlation coefficient $(r)$ of $-0.33 \pm 0.27$ after Fisher's z transformation and back-transformation (Chakraborty et al., 2020). This pattern is strongly controlled by availability of tree cover at the CBG scale (Fig. 7), because the presence of vegetation strongly controls the SUHI intensity (Fig. 6a) (Chakraborty and Lee, 2019; Zhou et al., 2016). Lower income CBGs have a lower percentage of tree cover in $\approx 69 \%$ (65 of 95) of cases $(r=0.17 \pm 0.3)$. Overall, the multi-city mean daytime summer SUHI is $1.95{ }^{\circ} \mathrm{C}$ for the highest income quartile CBGs and $2.74{ }^{\circ} \mathrm{C}$ for the lowest income quartile CBGs. Similarly, the multi-city mean annual canopy cover is $16.8 \%$ and $14.4 \%$ in the highest and lowest quartile of CBGs, respectively. 


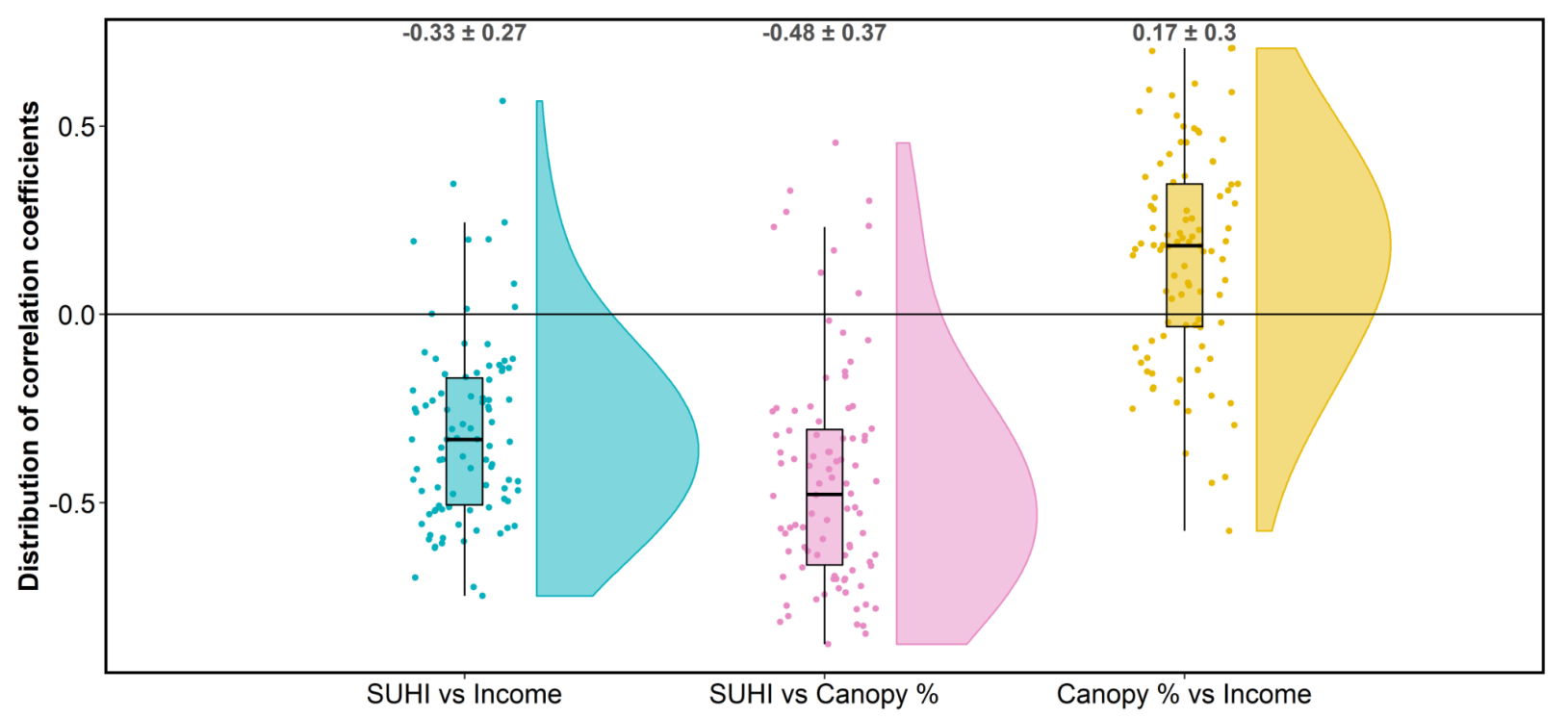

b
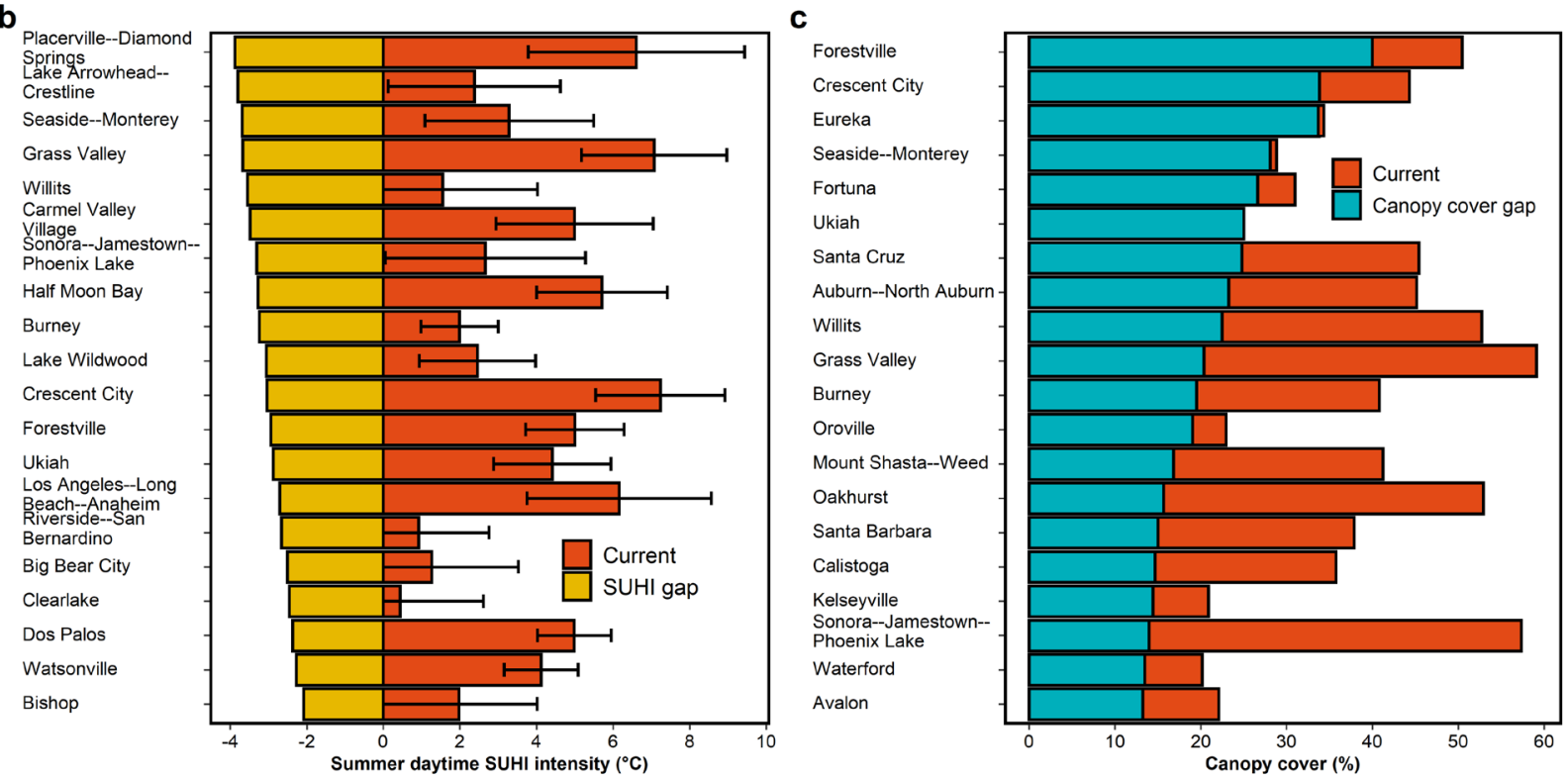

Fig. 6 Disparities in tree cover and urban heat islands across income groups. Sub-figure (a) shows the distribution of the correlation coefficient $(r)$ between CBG-level percentage tree canopy and median income, summer daytime surface urban heat island (SUHI) and percentage tree canopy, and SUHI and median income, respectively, across the cities with $>10$ CBGs. The composite mean correlations after Fisher's z transformation and back-transformation are also annotated. Random horizontal jitter is used to minimize overlap between points. Sub-figure (b) shows the 20 cities with the highest gap in summer daytime SUHI between the CBGs in the highest and lowest income quartiles, as well as the corresponding mean and standard deviation of the current SUHI intensity. Sub-figure (c) shows bar plots of the 20 cities with the highest gap in percentage tree canopy cover between the CBGs in the highest and lowest income quartiles, as well as the corresponding citylevel percentage tree canopy. 


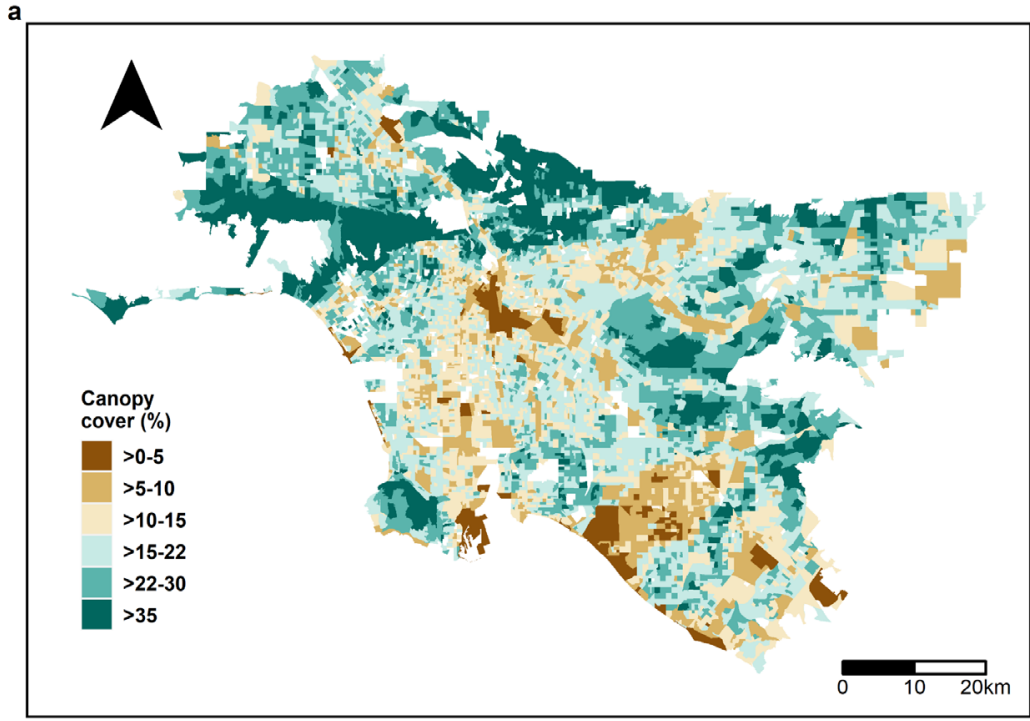

Fig. 7. Intra-urban variability in canopy cover. Sub-figures tree cover. 2005). To reiterate: urban forests. distribution of plantable area. between the highest income quartile and the urban CBGs.

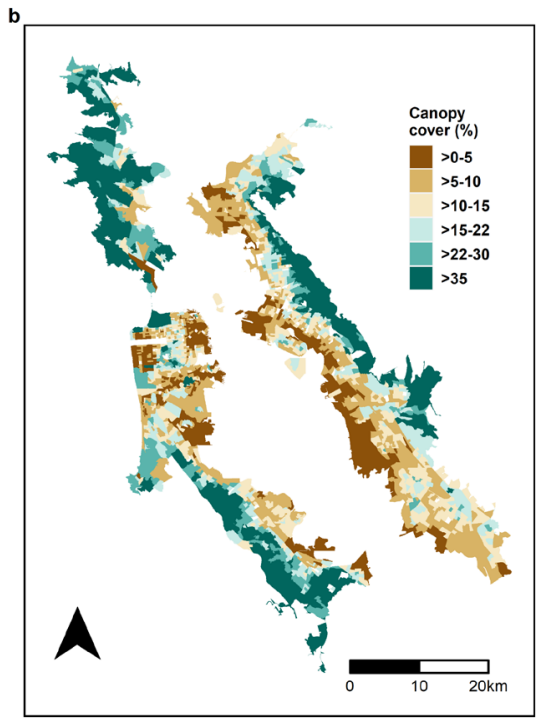

variability in canopy cover for San Francisco and Los Angeles, respectively, at the CBG level.

To illustrate further, we also calculate the difference in tree cover percentage and summer SUHI for the CBGs in the highest and lowest income quartile for each city. These can only be calculated for cities with at least 4 CBGs. Of the 166 cities that fulfill this criterion, 119 cities have a negative gap in summer daytime SUHI (i.e., CBGs in the highest quartile of income have a lower SUHI than those in the lowest quartile). Figure $6 \mathrm{~b}$ shows the 20 cities in California with the highest daytime summer SUHI and canopy cover gap, as well as their current city-wide mean SUHI and percentage

\subsection{Surface urban heat island mitigation through equitable urban afforestation scenarios}

As explained in more details in the methods section, we define multiple scenarios of urban afforestation at the CBG level by combining the NLCD (Wickham et al., 2021) and EarthDefine ("EarthDefine,") tree canopy cover data with census-derived estimates of income (Mather et al.,

- The MPUA scenario assumes complete afforestation in all of the plantable space within each urban CBG. This does not include converting parking lots or existing built-up areas to

- The TREEGAP scenario aims to close the disparity in tree cover while factoring in the

- The UHIGAP scenario targets the plantable area to close the daytime disparity in SUHI

Overall, Californian cities have space for 1.38 million acres of trees (MPUA scenario). To close the tree gap as much as possible (TREEGAP scenario), we would require 0.24 million acres of urban afforestation or 6.8 million additional trees. For cities that satisfy the statistical constraints for the UHIGAP scenario, the MPUA, TREEGAP, and UHIGAP scenarios yield 1.23 million, 0.23 million, and 0.6 million acres, respectively. The relatively small difference between this subset and the overall superset is caused by cities with CBGs less than or equal to 15 or the $r^{2}$ of the correlation between SUHI and tree cover percentage being less than 0.30 . These cover only $10 \%$ of the total area of cities considered (only $5.8 \%$ of the urban population). For the 73 cities that fulfill the criteria 
for inclusion, the MPUA scenario would reduce the summer daytime SUHI, and thus urban LST, by an average of $1.68{ }^{\circ} \mathrm{C}$, while the TREEGAP scenario would reduce it by an average of $0.3{ }^{\circ} \mathrm{C}$. Figure 8 shows both the available area for potential afforestation for the three scenarios for a subset of cities with the highest respective values, as well as the SUHI intensity for the different cases. Note that the daytime SUHI gap does not disappear for the corresponding scenario since most CBGs do not have enough plantable area to accommodate that acreage of afforestation. This potential lack of space availability is particularly an issue in poorer CBGs with more urban density and is an issue that is rarely focused on while discussing nature-based heat mitigation strategies in cities. Among the urban clusters shown in Fig. 8b, there are also cases (for instance, Sacramento, Riverside, etc.) where the daytime SUHI would be negative for the MPUA scenario. This suggests a large amount of plantable area for urban afforestation in those clusters or a large sensitivity of SUHI to canopy cover percentage (or both).

a

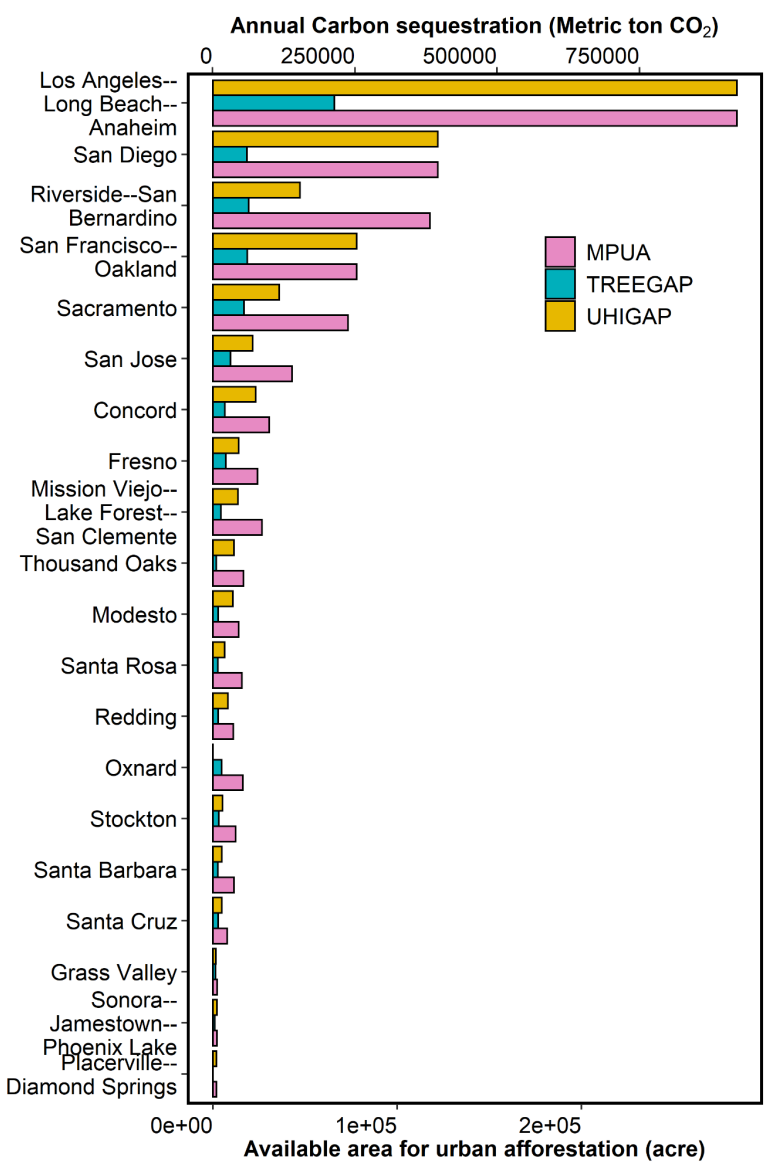

b

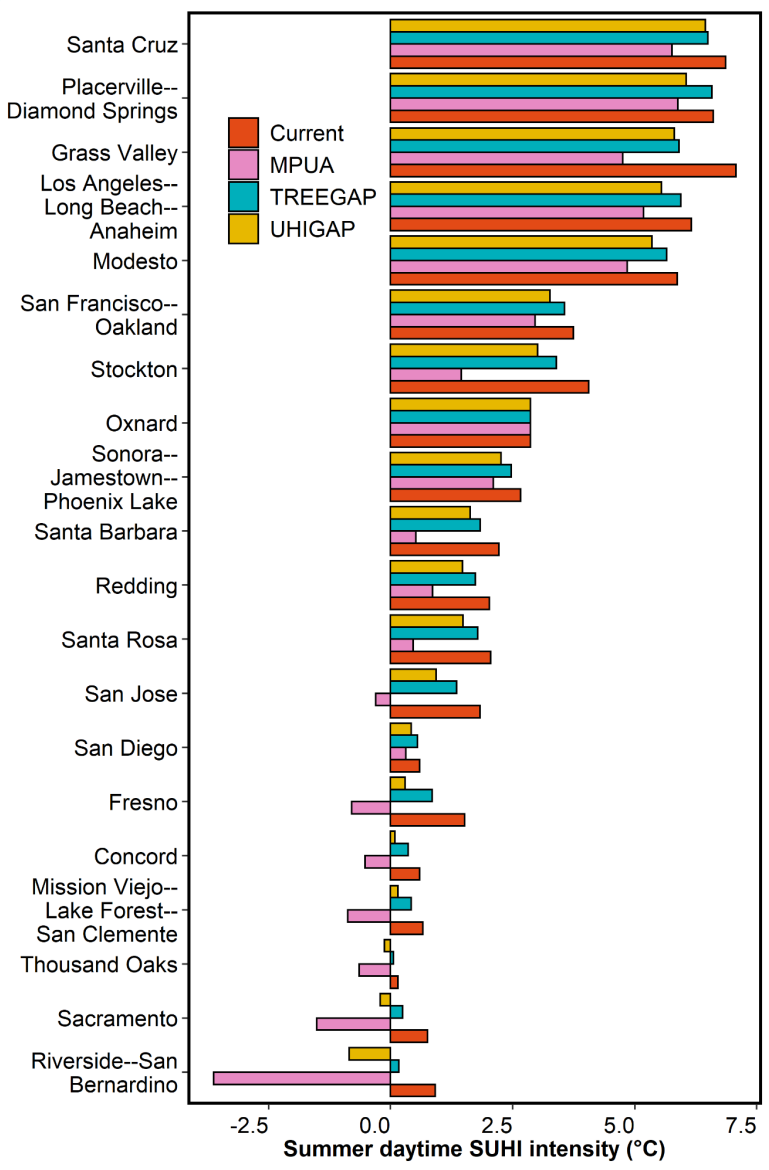

Fig. 8 Urban afforestation and associated SUHI mitigation. Sub-figure (a) shows the area available for urban afforestation for the MPUA, TREEGAP, and UHIGAP scenarios for the 20 cities with the largest current canopy area and at least 4 CBGs. The carbon sequestration for each scenario is on the top x axis. Sub-figure (b) shows the current average summer daytime SUHI, as well as the SUHI for the different afforestation scenarios for the 20 cities with the highest current SUHI intensity, number of CBGs greater than 15.

\subsection{Suitability scores for urban afforestation efforts}

Since funding and resources for tree planting and maintenance are limited, we developed a spatial prioritization algorithm that provides suitability scores at the CBG level (from 0 to 100) using two pathways - A1 and A2 - to close the gap in tree canopy cover and summer daytime SUHI between 
the highest income quartile CBGs and the CBGs of interest (Eqs 5 and 6). CBGs with higher A1 scores have more available area to close the tree canopy gap through afforestation, and are densely populated with lower income residents, thus benefiting a higher proportion of vulnerable people. A lower A1 score means that these blocks do not have enough space to completely close the disparity in tree cover from the highest quartile but will still benefit from closing the gap. Likewise, high A2 scores mean that these CBGs have enough space to reduce the disparity in summer daytime SUHI from high income CBGs of the city, which positively impacts vulnerable populations.

Out of 20,244 CBGs (21,358 CBGs for Californian cities) for which suitability scores could be calculated, 11,652 CBGs are suitable for afforestation through the A1 pathway while 3,402 of the rest are suitable for afforestation through pathway A2. The remaining CBGs are not suitable for afforestation within this framework because they either do not have enough available space to meet the tree cover needed to reduce SUHI intensity and/or do not benefit the lower income populations. The A1 CBGs equates to a total of 2.4 million acres, which is home to 21 million people, or about $57 \%$ of the urban population in California, and the A2 CBGs are home to almost 26 million people, accounting for an additional $14 \%$ of the population. If urban afforestation efforts are carried out across CBGs using both A1 and A2 pathways, we have an additional 853,396, 191,801, and 596,863 acres of urban trees for the MPUA, TREEGAP, and UHIGAP scenarios, respectively. This would reduce the current land area weighted mean daytime summer SUHI in these CBGs from $3.57^{\circ} \mathrm{C}$ to $1.83,2.53$, and $3.19^{\circ} \mathrm{C}$, respectively, for the three scenarios. Moreover, the CBGs targeted by this afforestation strategy contain almost $89 \%$ of the $\approx 9$ million urban residents in California in the lowest income quartile for their cities.

\subsection{Examining additional benefits and co-benefits}

The reduction in SUHI is a direct benefit of urban afforestation following this conceptual framework and allows one to also address disparities in potential heat exposure in cities. Urban tree cover however has several other direct and indirect benefits, ranging from increased carbon sequestration to reducing stormwater runoff to reducing heat-related mortality and morbidity. Drawing from estimates by McPherson et al. (McPherson et al., 2017, 2013), annual net carbon sequestration through afforestation would be 4.5, 0.8, and 2 million metric tons of $\mathrm{CO}_{2}$, respectively, under the MPUA, TREEGAP, and UHIGAP scenarios in California. This corresponds to net annual benefits ranging from $\$ 198$ million to $\$ 1.1$ billion ("Carbon Footprint Calculator Assumptions,"). Furthermore, afforestation in cities has the added benefit of leveraging the localscale benefits of green space, including urban heat mitigation, moderating air pollution concentrations, and reducing heat-related mortality and morbidity (Fargione et al., 2018; McDonald et al., 2020; Zhao et al., 2021). Figure 9 summarizes the multiple co-benefits that were examined during this study for multiple scenarios. 


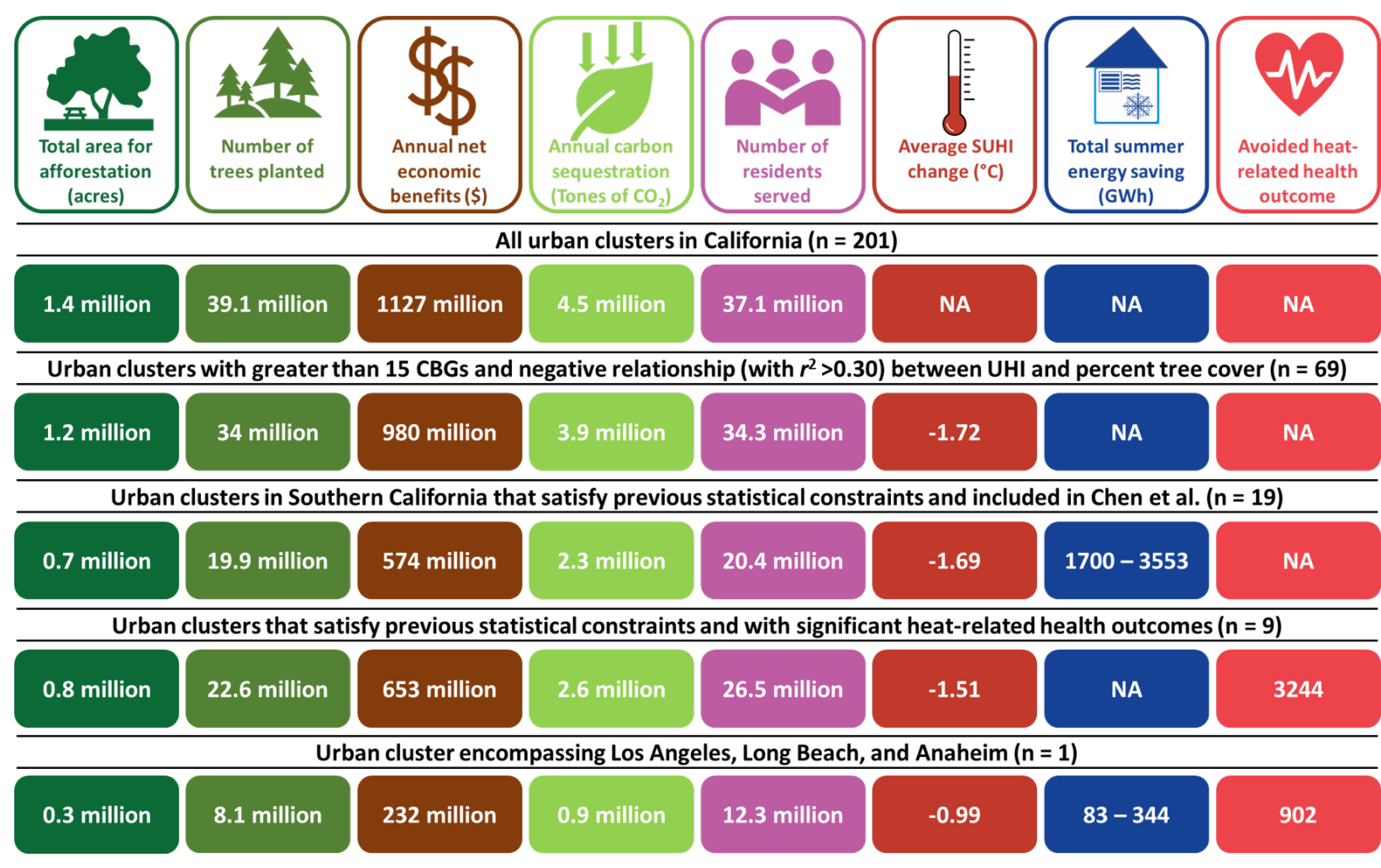

For 35 cities in southern California, where we had data on air-conditioning penetration rates and sensitivity of electricity consumption to ambient temperature (Chen et al., 2020), we also estimated reduction in cooling load due to urban afforestation. For the MPUA, TREEGAP, and UHIGAP scenarios, this translates to mean annual savings of $697 \mathrm{GWh}, 88 \mathrm{GWh}$, and $324 \mathrm{GWh}$, respectively (Fig. S2). These energy savings would reduce annual GHG emissions by approximately 166, 21, and 77 thousand metric tons (Bureau of Labor Statistics, US Department of Labor, 2019) (Fig. 9 shows a subset of results). The associated cost savings to residential users, assuming an average rate of $\$ 0.19$ per $\mathrm{kWh}$ (BLS reference), ranges from approximately $\$ 17$ to $\$ 132$ million per year, corresponding to the TREEGAP and MPUA scenarios, respectively (Table S1). These values are much higher than the monetary value of the GHG emissions reduction as reflected by the social cost of carbon (SCC), which would be valued at between $\$ 1.2$ million and $\$ 9.1$ million per year using the central estimate of $\$ 50$ per ton (Bureau of Labor Statistics, U.S. Department of Labor, 2019). Assuming a discount rate of $3 \%$, consistent with the central rate used by the US Interagency Working Group (Interagency Working Group, 2016), and linear canopy growth until reaching maturity in year 35, the net benefits over 40 years add up to approximately $\$ 186$ million for the TREEGAP and over 8 times more, approximately $\$ 1,481$ million, for the MPUA scenario (Table S2). In the absence of any intervention to reduce SUHI, the net present value of the social cost of carbon from residential electricity use ranges from $\$ 12.9$ million to $\$ 102.1$ million.

Finally, for a smaller subset of cities for which we had heat-related health outcome data, we estimate the avoidance of almost 4000 similar health outcomes for a corresponding 10-year period for the MPUA scenario (Fig. S1). Publicly available health related datasets are limited. Our health-related outcomes are summarized from heat-related mortality and morbidity data at zip code level for CA with more than 10 observations, which only included six cities. Overall, our analyses show that 
urban afforestation in California, while not having a strong impact on large-scale climate change mitigation and emission reduction goals (for instance, less than $0.2 \%$ of the US nationally determined contribution goals for 2030), would contribute to climate adaptation through urban heat mitigation and its associated local-scale benefits.

\section{Discussion and Conclusions}

California is experiencing a climate crisis with extensive heat waves during the summer with low income and vulnerable communities being disproportionately impacted (Shonkoff et al., 2011). Past studies have shown that across the US, low-income neighborhoods are hotter and have less tree cover than high income neighborhoods (Benz and Burney, 2021; Chakraborty et al., 2019; Hoffman et al., 2020; Hsu et al., 2021; McDonald et al., 2021; Nesbitt et al., 2019). In Los Angeles, for example, the lowest income quartile has $9.5 \%$ less canopy cover and $2.7^{\circ} \mathrm{C}$ higher LST than the highest income quartile based on our analysis. In the present study, we develop a scalable bottomup approach using satellite remote sensing, tree canopy cover data, and census estimates to address these disparities and strategically prioritize urban afforestation within a city by simultaneously closing the tree gap and reducing the surface urban heat island. Depending on the availability of funds and the costs of tree planting and maintenance, each city and local community can initiate a climate mitigation plan by first meeting the needs of the most impacted communities by closing the tree gap in $\mathrm{A} 1 \mathrm{CBGs}$, and follow this with additional intervention in the CBGs that can support further afforestation and potentially reduce the SUHI further (A2 CBGs). These findings are intended to inform policymakers and city planners with a suite of intentional options to logistically support future afforestation efforts within the state. It also provides decision makers a means to explore opportunities to secure resources through the public and private sector to realize the additional ecological benefits from urban afforestation.

A few considerations are necessary to contextualize the results of this study. First, our focus on satellite-derived LST allows for a spatially-explicit multi-city perspective that is difficult with ground-based observations of air temperature (Muller et al., 2013). We note that air temperature is more directly relevant to public health (Anderson et al., 2013; Venter et al., 2021) than LST. However, while the relationship between air temperature and tree canopy coverage may be somewhat different in strength than that between LST and tree cover, we expect the direction of these relationships to be similar (Novick and Katul, 2020). Tree cover can also reduce heat exposure and improve pedestrian comfort through its shading effect (Middel et al., 2021; Zhao et al., 2018), which is difficult to estimate using satellite observations. Second, it is evident that multiple strategies need to be combined for maximum local-scale heat mitigation. For cities, this includes surface albedo-based interventions such as reflective pavements and white roofs (Zhao et al., 2017). There are advantages and disadvantages of each. Although white roofs and reflective pavements are more efficient at heat mitigation than urban green space (Zhao et al., 2017), reflective pavements have also been found to increase radiant heat exposure for pedestrians (Taleghani et al., 2016). Third, with urban afforestation, a reduction in temperature would also be associated with increases in humidity, which may hinder the total impact on heat stress (Hass et al., 2016). Finally, our analysis only accounts for ground-level vegetation but several other forms of urban vegetation cover are possible (Wong et al., 2021). As such, our method for identifying potential areas for urban afforestation is intended to be used as a starting point for the planning, not as a siting tool.

Our suitability framework can be applied throughout the US and can be expanded to the rest of the world with the availability of high-resolution tree cover datasets (Hansen et al., 2013). Although satellite-derived products are generally spatially continuous after temporal compositing, our primary limitation when expanding these estimates to every single city would be the ground-based socioeconomic data needed to better estimate the disparities. For example, income data from the US census bureau are not publicly available below the CBG scale, thus precluding the estimation 
562 of income quartiles, and thus the tree and SUHI gaps, in small cities with fewer than 4 CBGs. 563 Similar socioeconomic information is even more difficult to consistently acquire in other countries 564 (Hsu et al., 2020). Similarly, if health related datasets are more readily available, our approach can 565 help us more accurately quantify the number of lives that can be saved and improved across the 566 nation through strategic intervention for each scenario. Although the adverse physiological impacts 567 of heat on human health is well established in the epidemiological literature, it is important to stress 568 that behavioral factors can also play an important role (Christidis et al., 2010; Hajat and Kosatky, 569 2010). These broad estimates of reductions in heat-related health outcomes due to urban 570 afforestation are meant to be indicative of the potential benefits of urban heat mitigation to further 571 support climate action. For other co-benefits of afforestation, including the economic and carbon 572 capture ones, it is important to stress that the numbers we draw from are based on broad-scale, 573 sometimes idealistic, assumptions; an issue that has been discussed extensively for global estimates 574 (Bastin et al., 2019; Grainger et al., 2019; Skidmore et al., 2019; Veldman et al., 2019).

575 Overall, our results indicate the necessity to establish more cross-sector collaborations and 576 engagement between public health, urban forestry, and utilities, to meet resources needed to mitigate climate impacts within cities that impact people disproportionately (Carter et al., 2015). With the recent popularity of tree planting projects such as Plant-for-the-Planet and the Trillion Tree Campaign (Goymer, 2018), cities have the opportunity to participate and secure funding for urban afforestation, which can benefit vulnerable populations. Our study quantifies several of these benefits, as well as co-benefits, and can be important for implementing equitable nature-based solutions in cities for climate change adaptation and mitigation. 


\section{Acknowledgements}

584 We would like to thank Paul English from Public Health Institute for providing us access to public 585 health data from the California Department of Public Health and Cheng et al., for sharing sensitivity 586 and air-conditioning penetration dataset for our study. We would also like to acknowledge Paul 587 English along with John Melvin from CalFire Urban Forestry and Jim Thorton from University of 588 California Davis for reviewing the initial draft of our paper. We would also like to thank Rob 589 McDonald from TNC for providing his guidance throughout the study and reviewing the final draft 590 of the paper. Lastly, this study could not have been possible without the generous support from 591 Matt Merrifield for supporting this research with the Conservation Technology Innovation Fund.

\section{Declaration of Competing Interests}

593 The authors declare that they have no competing financial interests.

\section{Data Statement}

595 The satellite data used here are publicly available on the Google Earth Engine Data Catalog 596 (https://developers.google.com/earth-engine/datasets). The EarthDefine data can be downloaded 597 from $h$ ttps://www.earthdefine.com/treemap/. The final suitability scores, as well as the intermediate 598 variables, are archived here: https://github.com/leahscampbell/CUTI-Scripts. The Census Block 599 Group level results can also be visualized through this Google Earth Engine web app: 600 https:/leahscampbell.users.earthengine.app/view/cuti-viewer. The scripts used to process the 601 satellite data and calculate the suitability scores can be accessed here: 602 https://github.com/leahscampbell/CUTI-Scripts 


\section{References}

2010 Census Urban and Rural Classification and Urban Area Criteria [WWW Document], n.d. URL \{https:/www.census.gov/programs-surveys/geography/guidance/geo-areas/urban-rural/2010-urbanrural.html\}

Abrams, M., 2000. The Advanced Spaceborne Thermal Emission and Reflection Radiometer (ASTER): data products for the high spatial resolution imager on NASA's Terra platform. Int. J. Remote Sens. 21, 847-859.

Anderson, G.B., Bell, M.L., Peng, R.D., 2013. Methods to Calculate the Heat Index as an Exposure Metric in Environmental Health Research. Environ. Health Perspect. 121, 1111-1119. https://doi.org/10.1289/ehp.1206273

Augusto, B., Roebeling, P., Rafael, S., Ferreira, J., Ascenso, A., Bodilis, C., 2020. Short and medium- to long-term impacts of nature-based solutions on urban heat. Sustain. Cities Soc. 57, 102122. https://doi.org/10.1016/j.scs.2020.102122

Bastin, J.-F., Finegold, Y., Garcia, C., Mollicone, D., Rezende, M., Routh, D., Zohner, C.M., Crowther, T.W., 2019. The global tree restoration potential. Science 365, 76-79. https://doi.org/10.1126/science.aax0848

Benz, S.A., Burney, J.A., 2021. Widespread Race and Class Disparities in Surface Urban Heat Extremes Across the United States. Earths Future 9. https://doi.org/10.1029/2021EF002016

Benz, S.A., Davis, S.J., Burney, J.A., 2021. Drivers and projections of global surface temperature anomalies at the local scale. Environ. Res. Lett. 16, 064093. https://doi.org/10.1088/1748-9326/ac0661

Bureau of Labor Statistics, U.S. Department of Labor, n.d. Average Energy Prices, Los Angeles-Long BeachAnaheim - May 2019 [WWW Document]. URL https://www.bls.gov/regions/west/newsrelease/2019/pdf/averageenergyprices_losangeles_20190617.pdf (accessed 6.6.21).

Carbon Footprint Calculator Assumptions [WWW Document], n.d. URL https:/www.pge.com/includes/docs/pdfs/about/environment/calculator/assumptions.pdf (accessed 6.6.21).

Carter, J.G., Cavan, G., Connelly, A., Guy, S., Handley, J., Kazmierczak, A., 2015. Climate change and the city: Building capacity for urban adaptation. Prog. Plan. 95, 1-66.

Chakraborty, T., Hsu, A., Manya, D., Sheriff, G., 2020. A spatially explicit surface urban heat island database for the United States: Characterization, uncertainties, and possible applications. ISPRS J. Photogramm. Remote Sens. 168, 74-88.

Chakraborty, T., Hsu, A., Manya, D., Sheriff, G., 2019. Disproportionately higher exposure to urban heat in lowerincome neighborhoods: a multi-city perspective. Environ. Res. Lett. 14, 105003. https://doi.org/10.1088/1748-9326/ab3b99

Chakraborty, T., Lee, X., 2019. A simplified urban-extent algorithm to characterize surface urban heat islands on a global scale and examine vegetation control on their spatiotemporal variability. Int. J. Appl. Earth Obs. Geoinformation 74, 269-280. https://doi.org/10.1016/j.jag.2018.09.015

Chakraborty, T., Sarangi, C., Lee, X., 2021. Reduction in human activity can enhance the urban heat island: insights from the COVID-19 lockdown. Environ. Res. Lett.

Chakraborty, T.C., Lee, X., Ermida, S., Zhan, W., 2021. On the land emissivity assumption and Landsat-derived surface urban heat islands: A global analysis. Remote Sens. Environ. 265, 112682.

Chen, M., Ban-Weiss, G.A., Sanders, K.T., 2020. Utilizing smart-meter data to project impacts of urban warming on residential electricity use for vulnerable populations in Southern California. Environ. Res. Lett. 15, 064001.

Christidis, N., Donaldson, G.C., Stott, P.A., 2010. Causes for the recent changes in cold-and heat-related mortality in England and Wales. Clim. Change 102, 539-553.

Cities must protect people from extreme heat, 2021. . Nature 595, 331-332. https://doi.org/10.1038/d41586-02101903-1

Danielson, J.J., Gesch, D.B., 2011. Global multi-resolution terrain elevation data 2010 (GMTED2010). US Department of the Interior, US Geological Survey.

Dorst, H., van der Jagt, A., Raven, R., Runhaar, H., 2019. Urban greening through nature-based solutions - Key characteristics of an emerging concept. Sustain. Cities Soc. 49, 101620. https://doi.org/10.1016/j.scs.2019.101620

Drescher, M., 2019. Urban heating and canopy cover need to be considered as matters of environmental justice. Proc. Natl. Acad. Sci. 116, 26153-26154.

Duguay-Tetzlaff, A., Bento, V.A., Göttsche, F.M., Stöckli, R., Martins, J., Trigo, I., Olesen, F., Bojanowski, J.S., Da Camara, C., Kunz, H., 2015. Meteosat land surface temperature climate data record: Achievable accuracy and potential uncertainties. Remote Sens. 7, 13139-13156.

EarthDefine [WWW Document], n.d. URL http://www.earthdefine.com/

Ermida, S.L., Soares, P., Mantas, V., Göttsche, F.-M., Trigo, I.F., 2020. Google earth engine open-source code for land surface temperature estimation from the landsat series. Remote Sens. 12, 1471.

Fargione, J.E., Bassett, S., Boucher, T., Bridgham, S.D., Conant, R.T., Cook-Patton, S.C., Ellis, P.W., Falcucci, A., Fourqurean, J.W., Gopalakrishna, T., others, 2018. Natural climate solutions for the United States. Sci. Adv. 4, eaat1869. 
Gallo, K.P., Tarpley, J.D., McNab, A.L., Karl, T.R., 1995. Assessment of urban heat islands: a satellite perspective. Atmospheric Res. 37, 37-43.

Gorelick, N., Hancher, M., Dixon, M., Ilyushchenko, S., Thau, D., Moore, R., 2017. Google Earth Engine: Planetaryscale geospatial analysis for everyone. Remote Sens. Environ. 202, 18-27.

Goymer, P., 2018. A trillion trees. Nat. Ecol. Evol. 2, 208-209.

Grainger, A., Iverson, L.R., Marland, G.H., Prasad, A., 2019. Comment on "The global tree restoration potential." Science 366, eaay8334. https://doi.org/10.1126/science.aay8334

Grimmond, C.S.B., Blackett, M., Best, M.J., Baik, J.-J., Belcher, S., Beringer, J., Bohnenstengel, S., Calmet, I., Chen, F., Coutts, A., others, 2011. Initial results from Phase 2 of the international urban energy balance model comparison. Int. J. Climatol. 31, 244-272.

Hajat, S., Kosatky, T., 2010. Heat-related mortality: a review and exploration of heterogeneity. J. Epidemiol. Community Health 64, 753-760.

Hansen, M.C., Potapov, P.V., Moore, R., Hancher, M., Turubanova, S.A., Tyukavina, A., Thau, D., Stehman, S., Goetz, S.J., Loveland, T.R., others, 2013. High-resolution global maps of 21 st-century forest cover change. science 342, 850-853.

Harlan, S.L., Brazel, A.J., Prashad, L., Stefanov, W.L., Larsen, L., 2006. Neighborhood microclimates and vulnerability to heat stress. Soc. Sci. Med. 63, 2847-2863.

Hass, A., Ellis, K., Reyes Mason, L., Hathaway, J., Howe, D., 2016. Heat and Humidity in the City: Neighborhood Heat Index Variability in a Mid-Sized City in the Southeastern United States. Int. J. Environ. Res. Public. Health 13, 117. https://doi.org/10.3390/ijerph13010117

Hoffman, J.S., Shandas, V., Pendleton, N., 2020. The Effects of Historical Housing Policies on Resident Exposure to Intra-Urban Heat: A Study of 108 US Urban Areas. Climate 8, 12. https://doi.org/10.3390/cli8010012

Hsu, A., Chakraborty, T., Thomas, R., Manya, D., Weinfurter, A., Chin, N., Goyal, N., Feierman, A., 2020. Measuring What Matters, Where It Matters: A Spatially Explicit Urban Environment and Social Inclusion Index for the Sustainable Development Goals. Front. Sustain. Cities 2, 556484.

Hsu, A., Sheriff, G., Chakraborty, T., Manya, D., 2021. Disproportionate exposure to urban heat island intensity across major US cities. Nat. Commun. 12, 2721. https://doi.org/10.1038/s41467-021-22799-5

Hulley, G.C., Dousset, B., Kahn, B.H., 2020. Rising trends in heatwave metrics across Southern California. Earths Future 8, e2020EF001480.

Imhoff, M.L., Zhang, P., Wolfe, R.E., Bounoua, L., 2010. Remote sensing of the urban heat island effect across biomes in the continental USA. Remote Sens. Environ. 114, 504-513.

Interagency Working Group, 2016. Technical support document: technical update of the social cost of carbon for regulatory impact analysis under executive order 12866.

Lewis, S.L., Maslin, M.A., 2015. Defining the Anthropocene. Nature 519, 171-180. https://doi.org/10.1038/nature14258

Liu, H., Huang, B., Zhan, Q., Gao, S., Li, R., Fan, Z., 2021. The influence of urban form on surface urban heat island and its planning implications: Evidence from 1288 urban clusters in China. Sustain. Cities Soc. 71, 102987. https://doi.org/10.1016/j.scs.2021.102987

Loveland, T.R., Dwyer, J.L., 2012. Landsat: Building a strong future. Remote Sens. Environ. 122, $22-29$.

Malakar, N.K., Hulley, G.C., Hook, S.J., Laraby, K., Cook, M., Schott, J.R., 2018. An operational land surface temperature product for Landsat thermal data: Methodology and validation. IEEE Trans. Geosci. Remote Sens. 56, 5717-5735.

Mather, M., Rivers, K.L., Jacobsen, L.A., 2005. The American community survey. Population Reference Bureau.

McDonald, R.I., Biswas, T., Sachar, C., Housman, I., Boucher, T.M., Balk, D., Nowak, D., Spotswood, E., Stanley, C.K., Leyk, S., 2021. The tree cover and temperature disparity in US urbanized areas: Quantifying the association with income across 5,723 communities. PLOS ONE 16, 1-27.

McDonald, R.I., Kroeger, T., Zhang, P., Hamel, P., 2020. The value of US urban tree cover for reducing heat-related health impacts and electricity consumption. Ecosystems 23, 137-150.

McMichael, A.J., Wilkinson, P., Kovats, R.S., Pattenden, S., Hajat, S., Armstrong, B., Vajanapoom, N., Niciu, E.M., Mahomed, H., Kingkeow, C., others, 2008. International study of temperature, heat and urban mortality: the 'ISOTHURM'project. Int. J. Epidemiol. 37, 1121-1131.

McPherson, E.G., Xiao, Q., Aguaron, E., 2013. A new approach to quantify and map carbon stored, sequestered and emissions avoided by urban forests. Landsc. Urban Plan. 120, 70-84.

McPherson, E.G., Xiao, Q., van Doorn, N.S., de Goede, J., Bjorkman, J., Hollander, A., Boynton, R.M., Quinn, J.F., Thorne, J.H., 2017. The structure, function and value of urban forests in California communities. Urban For. Urban Green. 28, 43-53.

Middel, A., Alkhaled, S., Schneider, F.A., Hagen, B., Coseo, P., 2021. 50 Grades of Shade. Bull. Am. Meteorol. Soc. 1-35. https://doi.org/10.1175/BAMS-D-20-0193.1

Muller, C.L., Chapman, L., Grimmond, C.S.B., Young, D.T., Cai, X., 2013. Sensors and the city: a review of urban meteorological networks: SENSORS AND THE CITY. Int. J. Climatol. 33, 1585-1600. https://doi.org/10.1002/joc.3678 
Nations, U., 2018. Revision of world urbanization prospects. U. N. N. Y. NY USA.

Nesbitt, L., Meitner, M.J., Girling, C., Sheppard, S.R., Lu, Y., 2019. Who has access to urban vegetation? A spatial analysis of distributional green equity in 10 US cities. Landsc. Urban Plan. 181, 51-79.

Novick, K.A., Katul, G.G., 2020. The duality of reforestation impacts on surface and air temperature. J. Geophys. Res. Biogeosciences 125, e2019JG005543.

Nowak, D.J., Greenfield, E.J., Hoehn, R.E., Lapoint, E., 2013. Carbon storage and sequestration by trees in urban and community areas of the United States. Environ. Pollut. 178, 229-236.

Oke, T.R., 1982. The energetic basis of the urban heat island. Q. J. R. Meteorol. Soc. 108, 1-24.

Remme, R.P., Frumkin, H., Guerry, A.D., King, A.C., Mandle, L., Sarabu, C., Bratman, G.N., Giles-Corti, B., Hamel, P., Han, B., Hicks, J.L., James, P., Lawler, J.J., Lindahl, T., Liu, H., Lu, Y., Oosterbroek, B., Paudel, B., Sallis, J.F., Schipperijn, J., Sosič, R., de Vries, S., Wheeler, B.W., Wood, S.A., Wu, T., Daily, G.C., 2021. An ecosystem service perspective on urban nature, physical activity, and health. Proc. Natl. Acad. Sci. 118, e2018472118. https://doi.org/10.1073/pnas.2018472118

Rouse, J.W., Haas, R.H., Schell, J.A., Deering, D.W., Harlan, J.C., 1974. Monitoring the vernal advancement and retrogradation (green wave effect) of natural vegetation. NASAGSFC Type III Final Rep. Greenbelt Md 371.

Santamouris, M., 2014. On the energy impact of urban heat island and global warming on buildings. Energy Build. $82,100-113$.

Shonkoff, S.B., Morello-Frosch, R., Pastor, M., Sadd, J., 2011. The climate gap: environmental health and equity implications of climate change and mitigation policies in California-a review of the literature. Clim. Change 109, 485-503.

Skidmore, A.K., Wang, T., de Bie, K., Pilesjö, P., 2019. Comment on "The global tree restoration potential." Science 366, eaaz0111. https://doi.org/10.1126/science.aaz0111

Taleghani, M., Sailor, D., Ban-Weiss, G.A., 2016. Micrometeorological simulations to predict the impacts of heat mitigation strategies on pedestrian thermal comfort in a Los Angeles neighborhood. Environ. Res. Lett. 11, 024003.

Veldman, J.W., Aleman, J.C., Alvarado, S.T., Anderson, T.M., Archibald, S., Bond, W.J., Boutton, T.W., Buchmann, N., Buisson, E., Canadell, J.G., Dechoum, M. de S., Diaz-Toribio, M.H., Durigan, G., Ewel, J.J., Fernandes, G.W., Fidelis, A., Fleischman, F., Good, S.P., Griffith, D.M., Hermann, J.-M., Hoffmann, W.A., Le Stradic, S., Lehmann, C.E.R., Mahy, G., Nerlekar, A.N., Nippert, J.B., Noss, R.F., Osborne, C.P., Overbeck, G.E., Parr, C.L., Pausas, J.G., Pennington, R.T., Perring, M.P., Putz, F.E., Ratnam, J., Sankaran, M., Schmidt, I.B., Schmitt, C.B., Silveira, F.A.O., Staver, A.C., Stevens, N., Still, C.J., Strömberg, C.A.E., Temperton, V.M., Varner, J.M., Zaloumis, N.P., 2019. Comment on "The global tree restoration potential." Science 366, eaay7976. https://doi.org/10.1126/science.aay7976

Venter, Z.S., Chakraborty, T., Lee, X., 2021. Crowdsourced air temperatures contrast satellite measures of the urban heat island and its mechanisms. Sci. Adv. 7, eabb9569. https://doi.org/10.1126/sciadv.abb9569

Voelkel, J., Hellman, D., Sakuma, R., Shandas, V., 2018. Assessing vulnerability to urban heat: A study of disproportionate heat exposure and access to refuge by socio-demographic status in Portland, Oregon. Int. J. Environ. Res. Public. Health 15, 640.

Wickham, J., Stehman, S.V., Sorenson, D.G., Gass, L., Dewitz, J.A., 2021. Thematic accuracy assessment of the NLCD 2016 land cover for the conterminous United States. Remote Sens. Environ. 257, 112357.

Wong, N.H., Tan, C.L., Kolokotsa, D.D., Takebayashi, H., 2021. Greenery as a mitigation and adaptation strategy to urban heat. Nat. Rev. Earth Environ. 2, 166-181. https://doi.org/10.1038/s43017-020-00129-5

Zhao, L., Lee, X., Schultz, N.M., 2017. A wedge strategy for mitigation of urban warming in future climate scenarios. Atmospheric Chem. Phys. 17, 9067-9080. https://doi.org/10.5194/acp-17-9067-2017

Zhao, Q., Guo, Y., Ye, T., Gasparrini, A., Tong, S., Overcenco, A., Urban, A., Schneider, A., Entezari, A., Vicedocabrera, A.M., others, 2021. Global, regional and national burden of mortality associated with non-optimal ambient temperatures from 2000 to 2019. Lancet Planet. Health.

Zhao, Q., Yang, J., Wang, Z.-H., Wentz, E.A., 2018. Assessing the cooling benefits of tree shade by an outdoor urban physical scale model at Tempe, AZ. Urban Sci. 2, 4.

Zheng, Z., Zhao, L., Oleson, K.W., 2021. Large model structural uncertainty in global projections of urban heat waves. Nat. Commun. 12, 1-9.

Zhou, D., Zhang, L., Li, D., Huang, D., Zhu, C., 2016. Climate-vegetation control on the diurnal and seasonal variations of surface urban heat islands in China. Environ. Res. Lett. 11, 074009.

Ziter, C.D., Pedersen, E.J., Kucharik, C.J., Turner, M.G., 2019. Scale-dependent interactions between tree canopy cover and impervious surfaces reduce daytime urban heat during summer. Proc. Natl. Acad. Sci. 116, 75757580. https://doi.org/10.1073/pnas.1817561116 